\title{
Temporal Dilation of Animal Cardiac Recordings Registered to Human Torso Geometries
}

\author{
Karli Gillette ${ }^{1}$, Jess Tate ${ }^{1}$, Brianna Kindall ${ }^{1}$, Wilson Good ${ }^{1}$, Jeff Wilkinson ${ }^{2}$, Narendra Simha ${ }^{2}$, \\ Rob MacLeod ${ }^{1}$ \\ ${ }^{1}$ SCI Institute, University of Utah, Salt Lake City, Utah, USA \\ ${ }^{2}$ Medtronic Inc., Minneapolis, Minnesota, USA
}

\begin{abstract}
Recordings of cardiac surface potentials from animal hearts can be mapped into human torso and used as source potentials for torso simulation. However, geometric registration of the heart can introduce changes in the effective conduction velocity due to change in relative positions of the recording sites. We developed a time dilation technique to ensure that adjusted cardiac potential recordings had physiological timing similar to human recordings after registration and corrected for conduction velocity.

Temporal dilation was performed both linearly and nonlinearly using two scaling techniques that reflect either global or local deformations. Linear temporal dilation of canine epicardial potential recordings using global scaling could be used to generate electrograms physiologically similar to humans in terms of conduction velocity, activation recovery interval, total activation time, and activation maps. Epicardial potential mapping of such dilated canine recordings thus allows the investigation of human-like arrhythmias and other disease states that can not be readily induced or measured in humans.
\end{abstract}

\section{Introduction}

Cardiac arrythymias are clinically detected, monitored, and treated using technologies like the 12-lead electrocardiogram (ECG) or an implantable cardioverter defibrillators (ICDs). The validation and development of such techniques, like testing new electrode placements and orientations, requires large databases of epicardial electrograms (EGs) for use within the ECG forward and inverse problems [1]. Due to the invasive nature of obtaining such EGs in humans, animal electrophysiological studies provide a valid alternative. We therefore developed a pipeline that utilized animal EGs mapped onto human hearts in the ECG forward problem called the cardiac arrhythmia potential simulation (caps) pipeline. The caps pipeline allows computational validation of ECGs, ICDs, or other compa- rable devices with a large database of pre-existing animal sinus and arrhythmias recordings.

The caps pipeline required overcoming inherent differences in physiology between animal and human hearts, and artifacts in propagation introduced by mapping animal hearts to humans. First, the smaller animal hearts have different time markers for the electrical properties of the heart, such as total activation time (TAT) and activation recovery interval (ARI). Second, spatially dependent parameters like conduction velocity (CV) are influenced when geometric spacing increases between EG recording locations on the epicardial surface, but the activation times remain constant. Important features within animal EGs, and subsequently ECGs obtained from the ECG forward problem, could become more physiology similar to humans with temporal correction.

Temporal dilation of animal cardiac recordings is proposed based on geometric deformation that occurs during geometric registration. In this study, two techniques of temporal dilation of canine EGs were investigated, namely linear and nonlinear. Linear resampled the entire signal uniformly, and nonlinear preserved the time derivatives in the QRS complex and T wave on a sinus signal while resampling all other segments. Both methods were based on the underlying assumption that $\mathrm{CV}$ should remain constant after mapping due to consistent cellular structure of the myocardium between canines and humans. To show physiological relevance following temporal, validation using conduction parameters was performed.

\section{Methods}

Ten sets of canine epicardial potential maps were attained from in situ, open-chest preparations using a sock of electrodes placed over the ventricles. Electrode socks consisted of either 128, 247, or 490 electrodes, where each sock was used for multiple experiments. Sock geometries were determined by digitizing recording sites on the electrode sock. Each EG set contained at least one beat during normal sinus rhythm and one run of arrhythmia, ven- 
tricular fibrillation or tachycardia. EGs were baseline corrected and band-pass filtered [2]. Bad leads were automatically detected according to signal-to-noise ratios, amplitude, and neighborhood correlation within all 10 data sets [3]. After detection, each time step within the EG was interpolated using trilinear interpolation based on the nearest neighbors.

Two human torso geometries with tissue regions of bones, lungs, blood volume, and pericardial heart, for utilization in the ECG forward problem, were provided through collaborations. Torso \#1, a surface mesh, was attained from the ECGSim software [4] from Radboud University in Nijmegen, The Netherlands. A segmentation of Torso \#2 was attained from Harvard University and the Childrens' Hospital Boston [5,6]. Tetgen [7], SCIRun [8], and Cleaver2 [9] were used to process the torso geometries and generate computational meshes. All three canine cardiac sock geometries were registered to both hearts defined within the human torso geometries using a modified ICP algorithm [10]. The sock electrode points were then mapped onto the human epicardial surface using SCIRun.

Scaling factors for temporal dilation were calculated based on the geometric deformation of edges that occurred during registration of canine sock geometries into Torso $\# 1$ and \#2. Two different types of scaling factors were calculated to investigate both the global and local effects of deformation. The global scaling factor was found as mean change in edge lengths after registration (Eq. 1). Local scaling factors were calculated for every node in the sock geometry as the median change in edge length to only primary neighbors as defined in the neighborhood connectivity (Eq. 2). Both local and global scaling factors were found for each geometric combination.

$$
\begin{gathered}
g=\text { median }\left(\left.\frac{d(j)_{o}}{d(j)_{\text {reg }}}\right|_{j=1} ^{E}\right) \\
l(i)=\text { median }\left.\left(\left.\frac{d(k)_{o}}{d(k)_{\text {reg }}}\right|_{k=1} ^{P(i)}\right)\right|_{i=1} ^{N}
\end{gathered}
$$

$d_{o}$ : original edge length between nodes on sock geometry

$d_{r e g}$ : edge length between nodes on sock geometry after registration

$E$ : total number of edges in sock geometry

$P$ : total number of primary neighbors for given node

$N$ : total number of nodes in sock geometry

Temporal dilation was then performed to satisfy the condition that the activation and recovery times were the original values multiplied by the scaling factor, thus preserving $\mathrm{CV}$. The linear approach resampled the entire EG homogeneously over time by either the global or local scaling factors [11]. Linear temporal dilation was performed on both the sinus beat and arrhythmic runs in all 10 of the canine cardiac data sets, using both global and local scaling factors.
Nonlinear temporal dilation, on the other hand, preserved temporal derivatives of the QRS complex and $\mathrm{T}$ wave by dilating portions of the EG outside these segments. Here, the entire segment before the QRS complex was resampled by a ratio, $r_{1}$, and the segment between the QRS complex and $\mathrm{T}$ wave was resampled by a second ratio, $r_{2}$. These ratios were a function of the scaling factor, activation and recovery times, and the QRS complex and T wave (Equations 3 and 4). The last segment of the EG after the $\mathrm{T}$ wave was dilated by the original scaling factor.

$$
\begin{gathered}
r_{1}=\frac{A T_{o}(s)-Q R S_{\text {start }}}{A T_{o}-Q R S_{\text {start }}} \\
r_{2}=\frac{T_{o}(s)-Q T_{\text {start }}-A T_{o}(s)-Q R S_{\text {end }}}{T_{o}-Q T_{\text {start }}-A T_{o}-Q R S_{\text {end }}}
\end{gathered}
$$

s: scaling factor

$Q R S_{\text {start }}$ : original start of the QRS complex

$A T_{o}$ : original activation time

$T_{o}$ : original recovery time

$Q T_{\text {start }}$ : start of the T-wave

$Q R S_{\text {end }}$ : original end of the QRS complex

To remove artifacts resulting from resampling while maintaining the integrity of the signal, each section was resampled with an additional five data points on each end and then cropped by a respective amount after dilation. All segments were then concatenated to reconstruct the entire EG. Nonlinear temporal dilation, using both local and global scaling, was performed on only the sinus beats within the ten canine data sets, due to the lack of the QRS complex and T-wave during arrhythmia.

Before temporal dilation, morphology characteristics of $\mathrm{CV}$, activation recovery interval (ARI), and total activation time (TAT) for each sinus beat were found across all $10 \mathrm{ca}-$ nine cardiac data sets. The temporally dilated canine data sets were evaluated for corresponding characteristics. All metrics, before and after dilation, were compared to human and canine values found in the literature. For qualitative analysis, activation maps were generated and compared to activation patterns in human ventricles.

\section{Results}

The mean of local scaling factors for each geometric combination of each canine sock geometry to human torso were comparable to the respective global scaling factors (Table 1). Local scaling factors exhibited the strongest deviation from the mean at the apex and basal regions of the ventricles, where deviation increased with increasing nodal size in the sock geometries.

Linear and nonlinear temporal dilation resulted in comparable activation and recovery times during sinus, but nonlinear temporal dilation resulted in a preserved temporal derivative in the QRS complex and T wave (Figure 1). Before temporal dilation, the mean CV, TAT, and ARI 
Table 1: Global and mean local scaling factors for 128 , 247 , and 490 sock electrode geometries when registered to hearts of Torso \#1 and Torso \#2.

\begin{tabular}{crcc}
\hline Sock Geometry & 128 & 247 & 490 \\
\hline \hline & \multicolumn{4}{c}{ Global Scaling Factor } \\
Torso \#1 & 1.48 & 1.64 & 1.57 \\
Torso \#2 & 1.64 & \multicolumn{3}{c}{ Local Scaling Factors } \\
& \multicolumn{4}{c}{1.71} \\
Torso \#1 & $1.49 \pm 0.01$ & $1.66 \pm 0.01$ & $1.58 \pm 0.01$ \\
Torso \#2 & $1.62 \pm 0.01$ & $1.87 \pm 0.01$ & $1.73 \pm 0.01$ \\
\hline \hline
\end{tabular}

for all 10 canine cardiac data sets was $33.41 \pm 3.19 \mathrm{~cm} / \mathrm{s}$, $165.40 \pm 12.64 \mathrm{~ms}$, and $33.60 \pm 3.94 \mathrm{~ms}$, respectively. Temporal dilation using global scaling factors resulted in the maintenance of $\mathrm{CV}$, while increasing both TAT and ARI in proportion to the scaling factor (Table 2). Temporal dilation using local scaling factors, however, resulted in lowered CVs and extended TATs, but a maintenance of the ARI (Table 2). Both implementations of temporal dilation performed similarly when comparing CV, TAT, and ARI.

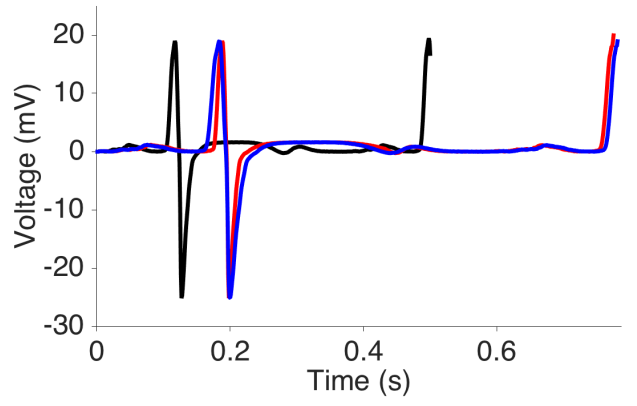

Figure 1: Linear (blue) and nonlinear (red) temporal dilation of canine epicardial EG during sinus (black).

Temporal dilation with global scaling factors resulted in the general maintenance of activation maps, though the range was proportionally scaled (Figures 2c). Local scaling, on the other hand, lead to visually different activation maps with a much larger range (Figure 2e). Though only linear dilation is shown, nonlinear was comparable in terms of activation maps, but with earlier activation. Similar results were also attained from the second torso.

\section{Discussion}

This study shows the feasibility of using temporal dilation to overcome differences in temporal electrical markers when using animal cardiac recordings in human torsos. Temporally dilated animal EGs, which more closely resemble humans, can be utilized in lieu of human data within the caps pipeline for the validation and development of technologies for cardiac arrhythmias.
Table 2: Mean CVs, ARIs, and TATs for all 10 canine cardiac data sets after temporal dilation using both local and global scaling factors.

\begin{tabular}{|c|c|c|c|}
\hline & $\mathrm{CV}(\mathrm{cm} / \mathrm{s})$ & ARI (ms) & TAT (ms) \\
\hline & \multicolumn{3}{|c|}{$\overline{\text { Linear-Global Scaling Factor }}$} \\
\hline Torso \#1 & $34.23 \pm 3.34$ & $269.86 \pm 25.58$ & $58.10 \pm 6.59$ \\
\hline \multirow[t]{2}{*}{ Torso \#2 } & $34.67 \pm 3.38$ & $250.82 \pm 1.29$ & $53.50 \pm 6.04$ \\
\hline & \multicolumn{3}{|c|}{ Nonlinear-Global Scaling Factor } \\
\hline Torso \#1 & $34.79 \pm 3.58$ & $280.67 \pm 23.62$ & $59.00 \pm 6.52$ \\
\hline Torso \#2 & $35.24 \pm 2.85$ & $252.67 \pm 21.53$ & $52.30 \pm 6.05$ \\
\hline & \multicolumn{3}{|c|}{ Linear-Local Scaling Factors } \\
\hline Torso \#1 & $17.34 \pm 3.12$ & $271.06 \pm 23.53$ & $265.70 \pm 41.84$ \\
\hline \multirow[t]{2}{*}{ Torso \#2 } & $18.93 \pm 2.95$ & $237.19 \pm 23.33$ & $192.10 \pm 24.08$ \\
\hline & \multicolumn{3}{|c|}{ Nonlinear-Local Scaling Factors } \\
\hline Torso \#1 & $17.33 \pm 3.14$ & $283.09 \pm 23.77$ & $268.70 \pm 42.12$ \\
\hline Torso \#2 & $18.61 \pm 2.64$ & $250.82 \pm 1.29$ & $193.40 \pm 24.36$ \\
\hline
\end{tabular}

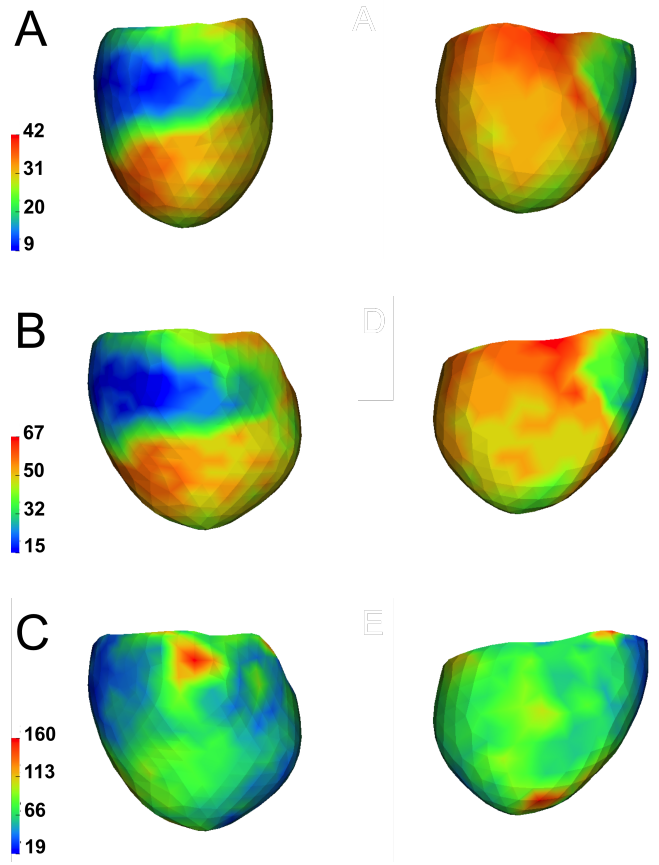

Figure 2: Activation maps for a single canine cardiac data set with a 490 electrode sock geometry, registered to Torso \#1, before (A) and after linear temporal dilation using either global (B) or local scaling factors (C). Respective scaling for each subfigure is in milliseconds.

Linear temporal dilation provided the only realistic option for the correction of canine EGs following registration of the canine heart into the human torso geometry. While nonlinear and linear techniques performed similarly in terms of CV, TAT, and ARI (Table 2), nonlinear temporal dilation can not be implemented on arrhythmic canine EGs due to the lack of morphological features like the QRS complex and $\mathrm{T}$ wave. This limitation makes nonlinear di- 
lation unviable in the context of cardiac arrhythmias, but allows possibility of maintaining temporal derivatives of important transient features in EGs.

Temporal dilation, both linear and nonlinear, using global scaling resulted in physiologically relevant EGs for humans in terms of CV and ARI (Table 2) $[15,17]$. Both the original and globally dilate signals (both linear and non-linear) had TATs slightly higher than expected, showing that the global dilation technique can also reflect changes in the original data $[14,16]$. Human-like activation patterns were generated using temporal dilation with global scaling, though comparison was based on a limited human studies on ventricular activation [18].

Temporal dilation with local scaling produced low CVs and abnormally high TATs for humans (Table 2) $[15,16]$. A primary explanation that the local scaling failed to properly represent local geometric deformations as intended was that propagation direction was not maintained, as indicated in differences between activation maps (Figure 2).

We have shown that some forms of temporal dilation can effectively modulate animal cardiac recordings to make them more physiologically comparable to human data for use in our caps pipeline. We were only able to compare values for sinus rhythm data, therefore further work could include validation of arrhythmic EGs. Such validation could be performed using simulation software like the Cardiac Arrhythmia Research Package [19] Additionally, further refinement of activation and recovery time detection, and development of a new method of local scaling could improve the dilation techniques and make it more robust.

\section{Acknowledgements}

This research was funded by Medtronic Inc.

\section{References}

[1] MacLeod R, Buist M. The forward problem of electrocardiography. In Macfarlane P, van Oosterom A, Pahlm O, Kligfield P, Janse M, Camm J (eds.), Comprehensive Electrocardiology. Springer Verlag, 2010; .

[2] Shome S, Stinstra J, Henriquez C, MacLeod R. Influence of extracellular potassium and reduced extracellular space on conduction velocity during acute ischemia: a simulation study. Journal of Electrocardiology 2006;39(4):S84-S85.

[3] Fisher R. Statistical methods for research workers 13th edn (edinburgh: Oliver and boyd) 1958;

[4] Van Oosterom A. Genesis of the $t$ wave as based on an equivalent surface source model. Journal of electrocardiology 2001;34(4):217-227.

[5] Jolley M, Stinstra J, Pieper S, MacLeod R, Brooks DH, Cecchin F, Triedman JK. A computer modeling tool for comparing novel icd electrode orientations in children and adults. Heart Rhythm 2008;5(4):565-572.
[6] Jolley M, Stinstra J, Tate J, Pieper S, MacLeod R, Chu L, Wang P, Triedman JK. Finite element modeling of subcutaneous implantable defibrillator electrodes in an adult torso. Heart Rhythm 2010;7(5):692-698.

[7] Si H, Gaertner K. Meshing piecewise linear complexes by constrained delaunay tetrahedralizations. In Proceedings of the 14th International Meshing Roundtable. 2005; 147-163.

[8] MacLeod R, Weinstein D, de St. Germain JD, Brooks D, Johnson C, Parker S. SCIRun/BioPSE: Integrated problem solving environment for bioelectric field problems and visualization. In IEEE International Symposium on Biomedical Imaging (ISBI). IEEE, IEEE Press, 2004; .

[9] CIBC, 2015. Cleaver: A MultiMaterial Tetrahedral Meshing Library and Application. Scientific Computing and Imaging Institute (SCI), Download from: http://www.sci.utah.edu/cibc/software.html.

[10] Chetverikov D, Svirko D, Stepanov D, Krsek P. The trimmed iterative closest point algorithm. In Pattern Recognition, 2002. Proceedings. 16th International Conference on, volume 3. IEEE, 2002; 545-548.

[11] Rabiner LR. Multirate digital signal processing. Prentice Hall PTR, 1996.

[12] Roberts DE, Hersh LT, Scher AM. Influence of cardiac fiber orientation on wavefront voltage, conduction velocity, and tissue resistivity in the dog. Circulation Research 1979; 44(5):701-712.

[13] Haws CW, Lux RL. Correlation between in vivo transmembrane action potential durations and activation-recovery intervals from electrograms. effects of interventions that alter repolarization time. Circulation 1990;81(1):281-288.

[14] Hill JD, Moore EN. Epicardial excitation studies in dogs with congenital right ventricular hypertrophy. Circulation research 1967;20(6):649-657.

[15] Katz AM. Physiology of the Heart. Lippincott Williams \& Wilkins, 2010.

[16] Cassidy DM, Vassallo JA, Marchlinski FE, Buxton AE, Untereker WJ, Josephson ME. Endocardial mapping in humans in sinus rhythm with normal left ventricles: activation patterns and characteristics of electrograms. Circulation 1984;70(1):37-42.

[17] Yue AM, Paisey JR, Robinson S, Betts TR, Roberts PR, Morgan JM. Determination of human ventricular repolarization by noncontact mapping validation with monophasic action potential recordings. Circulation 2004; 110(11):1343-1350.

[18] Durrer D, Van Dam RT, Freud G, Janse M, Meijler F, Arzbaecher R. Total excitation of the isolated human heart. Circulation 1970;41(6):899-912.

[19] Vigmond EJ, Hughes M, Plank G, Leon LJ. Computational tools for modeling electrical activity in cardiac tissue. Journal of electrocardiology 2003;36:69-74.

Address for correspondence:

Karli Gillette

SCI Institute-University of Utah, 72 Central Campus Dr, Salt Lake City, UT 84112

karli.gillette@gmail.com 